\title{
Efeito da Palhada de Cultivares de Cana-de-Açúcar na EMERGÊNCIA DE Cyperus rotundus ${ }^{1}$
}

\author{
Effects of Sugarcane Cultivars Straw on Cyperus rotundus Emergence
}

SILVA, J.R.V. ${ }^{2}$, COSTA, N.V. ${ }^{2}$ e MARTINS, D. ${ }^{3}$

\begin{abstract}
RESUMO - O objetivo deste estudo foi avaliar a emergência de plantas de tiririca (Cyperus rotundus) sob diferentes quantidades de palha de cana-de-açúcar posicionadas na superfície do solo. Foram testadas quantidades de palha equivalentes a $0,2,4,8,16$ e $20 \mathrm{t} \mathrm{ha}^{-1}$, das variedades de cana RB 82-5336 e SP 79-2233. Observou-se que a testemunha sem palha foi a que apresentou maior quantidade de plantas emersas. Os tratamentos com 2,4 e 8 t ha $^{-1}$ apresentaram comportamento semelhante ao da testemunha a partir dos 46 dias após o plantio. Os tratamentos com 16 e $20 \mathrm{t} \mathrm{ha}^{-1}$ de palha proporcionaram, durante todo o período experimental, menor quantidade de plantas emersas; contudo, em relação à biomassa seca da parte aérea, o tratamento com $20 \mathrm{t} \mathrm{ha}^{-1}$ comportou-se de forma semelhante à da testemunha. Não houve efeito das quantidades de palha testadas sobre o número de tubérculos produzidos. Os efeitos resultantes da palhada dos diferentes cultivares de cana-de-açúcar foram semelhantes.
\end{abstract}

Palavras-chave: palhada, tiririca, planta daninha.

\begin{abstract}
The objective of this study was to evaluate the emergence of purple nutsedge (Cyperus rotundus), under different amounts of sugar cane straw displaced on the soil surface. The treatments consisted of quantities of straw equivalent to $0,2,4,8,16$ and 20 tha $^{-1}$ from the cultivars RB 82 - 5336 and SP 79 - 2233. Control treatment without straw showed the largest number of emerged plants. The treatments with 2,4 and $8 \mathrm{tha}^{-1}$ had a behavior similar to the control, 46 days after planting. The treatments using 16 and $20 t \mathrm{ha}^{-1}$ of straw provided a smaller number of emerged plants throughout the experiment; however, the treatment with $20 \mathrm{tha}^{-1}$ was similar to the control, regarding shoot dry weight. No effect of the amounts of straw was observed on the number of tubers produced. The effects of different sugarcane cultivar straws were similar.
\end{abstract}

Key words: sugarcane straw, purple nutsedge, weed.

\section{INTRODUÇÃO}

As queimadas na cultura da cana-deaçúcar previamente à colheita são práticas usuais para facilitar o corte por meio manual. Entretanto, devido às pressões de cunho ambiental que o setor vem sofrendo por parte da sociedade pela melhoria da qualidade do ar nas cidades circunvizinhas a áreas canavieiras, segundo um esquema de restrições legais progressivas, até o ano de 2012, a queimada da cana-de-açúcar será proibida no Estado de São
Paulo (Decreto - lei no 41.719 de 16/07/97 e no 45.056 de 17/09/97). Dessa maneira, a palha após o corte deverá permanecer sobre o solo (Martins et al., 1999).

Em termos agronômicos, podem-se citar algumas vantagens da utilização dessa cobertura morta, como: aumento e manutenção do grau de umidade do solo, incremento da eficácia do controle de erosão, elevação da quantidade de matéria orgânica no solo, redução da população de nematóides nocivos

1 Recebido para publicação em 6.5.2003 e na forma revisada em 12.12.2003.

2 Eng.-Agrônomo, aluno de pós-graduação, Dep. de Produção Vegetal/Agricultura - FCA-UNESP, <jrvsilva@ fca.unesp.br>. ${ }^{3}$ Prof. Dr., Dep. de Produção Vegetal/Agricultura - FCA-UNESP, Caixa postal 237, 18603-970, Botucat-SP. 
e não-destruição dos inimigos naturais da broca, além da diminuição ou até eliminação do uso de herbicidas.

A quantidade, a composição, a periodicidade da produção e o tempo de permanência da cobertura morta numa área de cana-deaçúcar são características que dependem do cultivar, clima e manejo da área e devem determinar a composição da flora infestante e a eficiência do controle das plantas daninhas (Almeida, 1983; Almeida et al., 1983; Almeida \& Rodrigues, 1985). A adição da cobertura morta ao agroecossistema da cana-de-açúcar pode modificar a relação de plantas daninhas presentes na área, passando por significativas transformações fitossociológicas.

O efeito da cobertura morta no controle da comunidade infestante vem sendo atribuído à liberação de compostos alelopáticos e a efeitos físicos (Almeida \& Rodrigues, 1985). Os efeitos físicos da cobertura morta na comunidade infestante podem ser atribuídos à filtragem da luz, alterando a quantidade e a qualidade do comprimento das ondas luminosas e a manutenção da temperatura com menores oscilações (Taylorson \& Borthwick, 1969; Fener, 1980).

Martins et al. (1999), trabalhando com diferentes quantidades de cobertura morta com palha de cana-de-açúcar, do cultivar RB 825336, relatam que Sida rhombifolia sob quantidades de palha iguais ou superiores a $6 \mathrm{t} \mathrm{ha}^{-1}$ diminuiu sua agressividade, enquanto as espécies invasoras Bidens pilosa, Euphorbia heterophylla e Ipomea grandifolia tendem a manter-se como plantas-problema com quantidade de palha até $15 \mathrm{t} \mathrm{ha}^{-1}$.

Este trabalho teve por objetivo avaliar os efeitos da cobertura do solo com dois cultivares de cana-de-açúcar sobre a emergência de Cyperus rotundus.

\section{MATERIAL E MÉTODOS}

O experimento foi instalado e conduzido no Núcleo de Pesquisas Avançadas em Matologia - NUPAM, Fazenda Experimental Lageado, pertencente à FCA/UNESP, campus de Botucatu, no Estado de São Paulo. Foram utilizadas caixas de fibra de amianto com as seguintes dimensões: 0,37 x 0,37 x 0,23 m. O solo utilizado para o estudo foi um Latossolo
Vermelho-Amarelo. Não foram utilizados fertilizantes ou corretivos, e as características químicas da terra eram: $\mathrm{pH}\left(\mathrm{CaCl}_{2}\right)=4,4 ; \mathrm{M} . \mathrm{O} .\left(\mathrm{g} \mathrm{dm}^{-3}\right.$ $=30,0) ; \mathrm{P}=6,0 \mathrm{mg} \mathrm{dm}^{-3} ; \mathrm{H}+\mathrm{Al}, \mathrm{K}, \mathrm{Ca}, \mathrm{Mg}, \mathrm{SB}$ e CTC = 34,0;2,4;12,0;7,0;21,0 $\mathrm{mmol}_{\mathrm{c}} \mathrm{dm}^{-3}$, respectivamente; e $\mathrm{V} \%=82$.

O solo utilizado foi previamente peneirado e isento de qualquer outro tubérculo de tiririca. Todas as caixas foram preenchidas com a mesma quantidade de solo. O experimento foi disposto no delineamento inteiramente casualizado, com cinco repetições. Testaram-se os seguintes tratamentos: $0,2,4,8,16$ e $20 \mathrm{t} \mathrm{ha}^{-1}$ de palha seca de dois cultivares de cana-deaçúcar (RB 82-5336 e SP 79-2233). Os tubérculos de tiririca utilizados no estudo apresentavam-se viáveis, sendo tal fato determinado pela sua turgidez.

A palha utilizada no estudo para ambos os cultivares foi coletada logo após o corte mecânico da cultura (segundo corte). As plantas de tiririca (Cyperus rotundus) foram coletadas em área de cana-de-açúcar, com o auxílio de enxadas, nos primeiros 15 centímetros de solo; depois foram cortadas a parte aérea e as raízes e separados os tubérculos. Os tubérculos, em número de 75 , foram previamente contados, pesados para padronização do tamanho e colocados de maneira uniforme dentro das caixas. A profundidade de plantio foi de $5,0 \mathrm{~cm}$. Após o plantio dos tubérculos, o solo foi coberto com as respectivas quantidades de palha, para atingir os tratamentos propostos, e também com uma tela de arame a 5,0 centímetros de altura, a fim de evitar perda da palha pela ação do vento. Foram realizadas irrigações das caixas sempre que necessário. Na Tabela 1 são apresentados os dados de temperatura e precipitação do local vigentes durante a condução do experimento.

O número de plantas emersas nos tratamentos foi avaliado aos 11, 15, 18, 21, 25, 30, $36,46,59,72$ e 90 dias após o plantio (DAP). Foram consideradas emersas as plantas visíveis em cada avaliação e com mais de 1,0 centímetro de parte aérea, ainda que dentro da camada de palha. As avaliações foram encerradas aos 90 DAP, devido à estabilização da emergência de plantas. Na última avaliação, a camada de palha foi removida, seca e pesada. Com os valores dos pesos final (f) e inicial da palha (i), foram calculadas a quantidade de 
palha decomposta (d), pela fórmula $d=i-f$, e a taxa de decomposição (Td), pela fórmula: Td $(\%)=100 \mathrm{~d} / \mathrm{i}$. Para a quantificação da relação carbono/nitrogênio $(\mathrm{C} / \mathrm{N})$, o material foi previamente seco em estufa e a seguir moído, para análise química conforme metodologia de Tedesco et al. (1985). Foram avaliados também o número final de tubérculos, a biomassa seca final de tubérculos e a massa seca da parte aérea.

Os dados foram submetidos à análise de variância, utilizando níveis de probabilidade de $5 \%$; as médias foram comparadas com o auxílio do teste $\mathrm{t}$, a $5 \%$ de probabilidade.

Tabela 1 - Temperatura e precipitação médias durante o período de condução do estudo. Botucatu-SP, 2001

\begin{tabular}{|l|c|c|}
\hline \multicolumn{1}{|c|}{ Mês } & $\begin{array}{c}\text { Temperatura } \\
\left({ }^{\circ} \mathrm{C}\right)\end{array}$ & $\begin{array}{c}\text { Precipitação } \\
(\mathrm{mm})\end{array}$ \\
\hline 1 a 15 de setembro & 20,2 & 4,4 \\
\hline 16 a 30 de setembro & 16,9 & 1,4 \\
\hline 1 a 15 de outubro & 19,6 & 4,4 \\
\hline 16 a 31 de outubro & 20,5 & 5,1 \\
\hline 1 a 15 de novembro & 21,2 & 2,6 \\
\hline 16 a 30 de novembro & 22,4 & 5,2 \\
\hline 1 a 15 de dezembro & 20,4 & 11,1 \\
\hline
\end{tabular}

\section{RESULTADOS E DISCUSSÃO}

A cobertura morta no solo é responsável pela formação de microclima com temperatura, teor de umidade e população microbiana característica de cada tipo e quantidade de palha encontrada (Wisniewski \& Holtz, 1997). Cada variedade de cana apresenta normalmente uma relação $\mathrm{C} / \mathrm{N}$ diferente; quanto menor esse valor, pode-se supor que mais rapidamente ela irá se decompor, dependendo das condições do meio (Vigil \& Kissel, 1998). As relações $\mathrm{C} / \mathrm{N}$ para os dois cultivares de canade-açúcar estão expressas na Tabela 2.

Em termos absolutos, tanto a quantidade de palha decomposta como a taxa de decomposição foram menos intensas nos tratamentos que apresentavam menor quantidade de palha ( 2 e $4 \mathrm{t} \mathrm{ha}^{-1}$ ). Talvez esse fato possa ser explicado pela maior dificuldade em obter um ambiente favorável ao desenvolvimento da população microbiana (Brown \& Dickey, 1970), em razão das constantes oscilações térmicas, nos tratamentos com menores quantidades de palha. Observa-se também, em relação à taxa de decomposição, um valor menor para a quantidade de $4 \mathrm{t} \mathrm{ha}^{-1}$ - supõe-se que esse fato seja devido a uma comunidade de microrganismos ainda não totalmente formada e bastante dependente das condições ambientais (luz, temperatura e umidade), bem como da localização do material adicionado (Parker \& Larson, 1962).

A diferença da velocidade de decomposição entre os dois cultivares, com taxa maior para a variedade SP 79-2233, possivelmente se deveu à composição e quantidade de compostos, como lignina e celulose (Mason, 1980), encontradas em cada cultivar de cana-de-açúcar. Guanto à queda na taxa de decomposição nas condições de cobertura máxima (20 t ha-1) para ambos os cultivares, mas de forma mais intensa na variedade SP 79-2233, acredita-se que a população microbiana tenha atingido um patamar adequado à disponibilidade de matéria orgânica e que não foi suficiente para decompor com a mesma rapidez quantidades maiores, razão pela qual se estabilizou.

Distribuição ampla, dificuldade de controle e nocividade são fatores que interferem no manejo da tiririca. Têm-se observado que

Tabela 2 - Quantidade de palha inicial, final e decomposta sobre a superfície do solo, taxa de decomposição e relação C/N dos cultivares SP 792233 e RB 825336. Botucatu-SP, 2001

\begin{tabular}{|c|c|c|c|}
\hline \multicolumn{3}{|c|}{ Quantidade de palha $\left(\mathrm{t} \mathrm{ha}^{-1}\right)$} & Taxa de decomposição \\
\hline Inicial & Final & Decomposta & $(\%)$ \\
\hline \multicolumn{3}{|c|}{ SP 79 2233 } \\
\hline 2 & 1,67 & 0,33 & 16,5 \\
4 & 3,90 & 0,10 & 2,5 \\
8 & 6,63 & 1,37 & 17,2 \\
16 & 10,98 & 5,02 & 31,4 \\
20 & 14,56 & 5,44 & 27,2 \\
\hline \multicolumn{3}{|c|}{ RB 82 5336 } \\
\hline 2 & 1,82 & 0,18 & 9,2 \\
4 & 3,84 & 0,16 & 4,0 \\
8 & 7,48 & 0,52 & 6,5 \\
16 & 12,49 & 3,51 & 22,0 \\
20 & 15,84 & 4,16 & 20,8 \\
\hline \multicolumn{4}{|c|}{ RELAÇÃO C/N } \\
\hline SP 79 2233 & 49,3 & 52,6 \\
RB 825336
\end{tabular}

Planta Daninha, Viçosa-MG, v.21, n.3, p.375-380, 2003 
mesmo em áreas com colheita mecanizada, onde a tiririca fica sujeita a grandes quantidades de resíduos da cultura, ela continua ocorrendo com freqüência relativa elevada e com a maior importância relativa dentre as plantas daninhas infestantes.

As diferentes quantidades de palha de camadas testadas como cobertura vegetal foram capazes de reduzir o número de plantas emersas até os 30 DAP, no cultivar RB 82-5336 (Tabela 3) e até os 46 DAP no cultivar SP 792233 (Tabela 4). A partir desse período novas brotações emergiram, sendo utilizadas de $2 \mathrm{e}$ 4 t ha ${ }^{-1}$ de palha de ambos os cultivares - quantidades semelhantes à da testemunha. No final do período experimental, aos 90 DAP, para ambos os cultivares, somente quantidades elevadas de cobertura vegetal ( 16 e 20 t ha $^{-1}$ ) continuaram mostrando efeito supressor sobre a emergência de plantas.

O efeito supressor da cobertura morta observado na emergência de plantas com quantidades elevadas de palha ( 16 e $20 \mathrm{t} \mathrm{ha}^{-1}$ ) não foi verificado para biomassa seca da parte aérea das plantas, mostrando-se superior para a quantidade de palha de $20 \mathrm{t} \mathrm{ha}^{-1}$. Este tratamento também foi responsável pelo maior incremento de biomassa seca de tubérculos; ressalta-se ainda que o número de tubérculos encontrados foi semelhante entre as quantidades de palha testadas aos 90 DAP (Tabelas 5 e 6).

Tabela 3 - Número médio de plantas de tiririca emersas sob diferentes quantidades de palha do cultivar RB 825336. BotucatuSP, 2001

\begin{tabular}{|c|c|c|c|c|c|c|c|c|c|c|c|}
\hline \multirow{2}{*}{$\begin{array}{l}\text { Quantidade de } \\
\text { palha }\left(\mathrm{t} \mathrm{ha}^{-1}\right)\end{array}$} & \multicolumn{11}{|c|}{ Dias após o plantio (DAP) } \\
\hline & 11 & 15 & 18 & 21 & 25 & 30 & 36 & 46 & 59 & 72 & 90 \\
\hline 0 & $4,6 \mathrm{a}$ & $28,6 \mathrm{a}$ & $44,6 \mathrm{a}$ & $55,2 \mathrm{a}$ & $62,0 \mathrm{a}$ & $64,6 a$ & $65,0 \mathrm{a}$ & $68,4 \mathrm{a}$ & $76,8 \mathrm{ab}$ & $88,4 \mathrm{a}$ & $107,8 \mathrm{a}$ \\
\hline 2 & $0,0 \mathrm{~b}$ & $7,4 \mathrm{~b}$ & $18,6 \mathrm{~b}$ & $33,4 \mathrm{~b}$ & $48,4 \mathrm{~b}$ & $52,8 \mathrm{~b}$ & $61,8 \mathrm{ab}$ & $68,4 \mathrm{a}$ & $80,4 \mathrm{a}$ & $88,8 \mathrm{a}$ & $102,6 \mathrm{a}$ \\
\hline 4 & $0,0 \mathrm{~b}$ & $4,6 \mathrm{~b}$ & $11,6 \mathrm{bc}$ & $22,2 \mathrm{c}$ & $37,8 \mathrm{~b}$ & $41,4 \mathrm{bc}$ & $54 \mathrm{bc}$ & $52,6 \mathrm{ab}$ & $72,6 \mathrm{ab}$ & $81,0 \mathrm{ab}$ & $98,2 \mathrm{a}$ \\
\hline 8 & $0,0 \mathrm{~b}$ & $2,2 \mathrm{~b}$ & $6,6 \mathrm{~cd}$ & $11,6 \mathrm{~d}$ & $23,8 \mathrm{c}$ & $33,2 \mathrm{c}$ & $46,4 \mathrm{c}$ & $51,4 \mathrm{ab}$ & $63,0 \mathrm{~b}$ & $70,0 \mathrm{~b}$ & $92,4 \mathrm{ab}$ \\
\hline 16 & $0,0 \mathrm{~b}$ & $0,0 \mathrm{~b}$ & $0,8 \mathrm{~d}$ & $2,4 \mathrm{e}$ & $7,4 \mathrm{~d}$ & $13,6 \mathrm{~d}$ & $23,2 \mathrm{~d}$ & $39,4 \mathrm{~b}$ & $38,4 \mathrm{c}$ & $48,0 \mathrm{c}$ & $73,0 \mathrm{~b}$ \\
\hline 20 & $0,0 \mathrm{~b}$ & $0,0 \mathrm{~b}$ & $0,6 \mathrm{~d}$ & $1,6 \mathrm{e}$ & $7,0 \mathrm{~d}$ & $11,4 \mathrm{~d}$ & $21,4 \mathrm{~d}$ & $32,6 \mathrm{~b}$ & $40,2 \mathrm{c}$ & $46,8 \mathrm{c}$ & $72,2 \mathrm{~b}$ \\
\hline $\mathrm{F}_{\text {Tratamento }}$ & $12,02 *$ & $18,84 *$ & $28,65^{*}$ & $48,23^{*}$ & $37,98 *$ & $28,88^{*}$ & $27,99 *$ & $4,53^{*}$ & $12,08^{*}$ & $11,22 *$ & $4,68^{*}$ \\
\hline $\mathrm{CV}(\%)$ & 158,0 & 78,7 & 50,1 & 31,6 & 26,1 & 24,4 & 17,7 & 29,5 & 19,2 & 18,1 & 17,2 \\
\hline d.m.s. & 1,60 & 7,40 & 9,13 & 8,78 & 10,69 & 11,63 & 10,57 & 20,31 & 15,67 & 16,87 & 20,65 \\
\hline
\end{tabular}

Médias seguidas de mesma letra, na coluna, não diferem estatisticamente entre si pelo teste $\mathrm{t}(\mathrm{P}>0,05)$.

* Significativo a $5 \%$ de probabilidade.

Tabela 4 - Número médio de plantas de tiririca emersas sob diferentes quantidades de palha do cultivar SP 792233. BotucatuSP, 2001

\begin{tabular}{|c|c|c|c|c|c|c|c|c|c|c|c|}
\hline \multirow{2}{*}{$\begin{array}{l}\text { Quantidade de } \\
\text { palha }\left(\mathrm{t} \mathrm{ha}^{-1}\right)\end{array}$} & \multicolumn{11}{|c|}{ Dias após o plantio (DAP) } \\
\hline & 11 & 15 & 18 & 21 & 25 & 30 & 36 & 46 & 59 & 72 & 90 \\
\hline 0 & $7,4 \mathrm{a}$ & $35,2 \mathrm{a}$ & $53,8 \mathrm{a}$ & $61,6 \mathrm{a}$ & $71,8 \mathrm{a}$ & $70,4 \mathrm{a}$ & $67,8 \mathrm{a}$ & $78,8 \mathrm{a}$ & $87,4 \mathrm{a}$ & $95,4 a$ & $113,6 \mathrm{a}$ \\
\hline 2 & $0,2 \mathrm{~b}$ & $10,0 \mathrm{~b}$ & $27 \mathrm{~b}$ & $41,2 \mathrm{~b}$ & $57,2 \mathrm{~b}$ & $56,8 \mathrm{~b}$ & $56,0 \mathrm{~b}$ & $64,0 \mathrm{~b}$ & $83,4 \mathrm{a}$ & $93,2 \mathrm{ab}$ & $107,2 \mathrm{ab}$ \\
\hline 4 & $0,2 \mathrm{~b}$ & $8,6 \mathrm{~b}$ & $19,8 \mathrm{bc}$ & $30,8 \mathrm{c}$ & $46,0 \mathrm{c}$ & $45,4 \mathrm{c}$ & $49,8 \mathrm{bc}$ & $63,6 \mathrm{~b}$ & $77,6 \mathrm{ab}$ & $89,8 \mathrm{ab}$ & $101,4 \mathrm{ab}$ \\
\hline 8 & $0,0 \mathrm{~b}$ & $6,6 \mathrm{bc}$ & $14,0 \mathrm{~cd}$ & $21,6 \mathrm{~d}$ & $35,4 \mathrm{~d}$ & $41,0 \mathrm{c}$ & $41,6 \mathrm{c}$ & $58,2 \mathrm{~b}$ & $69,4 \mathrm{~b}$ & $80 \mathrm{bc}$ & $96,8 \mathrm{ab}$ \\
\hline 16 & $0,0 \mathrm{~b}$ & $1,0 \mathrm{c}$ & $5,4 \mathrm{de}$ & 8,4 e & $21,4 \mathrm{e}$ & $25,4 \mathrm{~d}$ & $28,6 \mathrm{~d}$ & $40,6 \mathrm{c}$ & $52,8 \mathrm{c}$ & $71,4 \mathrm{~cd}$ & $92,0 \mathrm{~b}$ \\
\hline 20 & $0,0 \mathrm{~b}$ & $1,0 \mathrm{c}$ & $3,8 \mathrm{e}$ & $5,0 \mathrm{e}$ & $14,6 \mathrm{e}$ & $21,2 \mathrm{~d}$ & $24,2 \mathrm{~d}$ & $39,2 \mathrm{c}$ & $50,0 \mathrm{c}$ & $63,2 \mathrm{~d}$ & $88,4 \mathrm{~b}$ \\
\hline $\mathrm{F}_{\text {Tratamento }}$ & $7,58^{*}$ & $25,54 *$ & $33,53 *$ & $64,72 *$ & $57,54 *$ & $31,29 *$ & $24,23 *$ & $10,78 *$ & $11,24^{*}$ & $8,22 *$ & $2,14^{*}$ \\
\hline $\mathrm{CV}(\%)$ & 186,84 & 54,13 & 34,52 & 21,05 & 15,56 & 17,17 & 16,87 & 18,02 & 14,96 & 12,27 & 14,46 \\
\hline d.m.s. & 3,2 & 7,4 & 9,4 & 7,8 & 8,4 & 9,8 & 9,9 & 13,7 & 13,8 & 13,3 & 19,1 \\
\hline
\end{tabular}

Médias seguidas de mesma letra, na coluna, não diferem estatisticamente entre si pelo teste $\mathrm{t}(\mathrm{P}>0,05)$.

* Significativo a $5 \%$ de probabilidade. 
Tabela 5 - Número e biomassa seca de tubérculos e biomassa seca da parte aérea de plantas de tiririca submetidas a diferentes quantidades de cobertura de palha de cana-deaçúcar do cultivar RB 82 5336, aos 90 DAP. Botucatu-SP, 2001

\begin{tabular}{|c|c|c|c|}
\hline $\begin{array}{c}\text { Quantidade de } \\
\text { palha }\left(\mathrm{t} \mathrm{ha}^{-1}\right)\end{array}$ & $\begin{array}{c}\text { Número de } \\
\text { tubérculos }\end{array}$ & $\begin{array}{c}\text { Biomassa seca } \\
\text { de tubérculos }\end{array}$ & $\begin{array}{c}\text { Biomassa seca } \\
\text { da parte aérea }\end{array}$ \\
\hline 0 & 236,20 & $72,10 \mathrm{~b}$ & $17,62 \mathrm{ab}$ \\
\hline 2 & 243,60 & $68,82 \mathrm{~b}$ & $15,25 \mathrm{~b}$ \\
\hline 4 & 263,20 & $83,62 \mathrm{ab}$ & $15,60 \mathrm{~b}$ \\
\hline 8 & 272,60 & $92,86 \mathrm{ab}$ & $15,98 \mathrm{ab}$ \\
\hline 16 & 214,40 & $82,87 \mathrm{ab}$ & $17,90 \mathrm{ab}$ \\
\hline 20 & 249,80 & $100,41 \mathrm{a}$ & $19,22 \mathrm{a}$ \\
\hline $\mathrm{F}_{\text {Tratamento }}$ & $1,07^{\mathrm{NS}}$ & $2,07^{*}$ & $1,99^{*}$ \\
\hline $\mathrm{CV}(\%)$ & 18,0 & 22,3 & 14,6 \\
\hdashline d.m.s. & 58,60 & 24,58 & 3,26 \\
\hline
\end{tabular}

Médias seguidas de mesma letra, na coluna, não diferem estatisticamente entre si pelo teste $\mathrm{t}(\mathrm{P}>0,05)$.

* Significativo a $5 \%$ de probabilidade.

${ }^{\text {NS }}$ Não-significativo a $5 \%$ de probabilidade.

Tabela 6 - Número e biomassa seca de tubérculos e biomassa seca da parte de plantas de tiririca submetidas a diferentes quantidades de cobertura de palha de cana-deaçúcar do cultivar SP 79 2233, aos 90 DAP. BotucatuSP, 2001

\begin{tabular}{|c|c|c|c|}
\hline $\begin{array}{c}\text { Quantidade de } \\
\text { palha }\left(\mathrm{t} \mathrm{ha}^{-1}\right)\end{array}$ & $\begin{array}{c}\text { Número de } \\
\text { tubérculos }\end{array}$ & $\begin{array}{c}\text { Biomassa seca } \\
\text { de tubérculos }\end{array}$ & $\begin{array}{c}\text { Biomassa seca } \\
\text { da parte aérea }\end{array}$ \\
\hline 0 & 195,40 & $67,42 \mathrm{~b}$ & $18,69 \mathrm{ab}$ \\
\hline 2 & 217,00 & $78,92 \mathrm{ab}$ & $17,51 \mathrm{~b}$ \\
\hline 4 & 218,60 & $71,40 \mathrm{ab}$ & $16,38 \mathrm{~b}$ \\
\hline 8 & 246,60 & $92,94 \mathrm{ab}$ & $17,59 \mathrm{~b}$ \\
\hline 16 & 228,00 & $82,65 \mathrm{ab}$ & $18,83 \mathrm{ab}$ \\
\hline 20 & 238,00 & $102,34 \mathrm{a}$ & $22,17 \mathrm{a}$ \\
\hline $\mathrm{F}_{\text {Tratamento }}$ & $0,80^{\mathrm{NS}}$ & $1,56^{*}$ & $1,80^{*}$ \\
\hline CV $(\%)$ & 19,0 & 28,5 & 17,9 \\
\hline d.m.s. & 56,30 & 31,10 & 4,38 \\
\hline
\end{tabular}

Médias seguidas de mesma letra, na coluna, não diferem estatisticamente entre si pelo teste $\mathrm{t}(\mathrm{P}>0,05)$.

* Significativo a $5 \%$ de probabilidade.

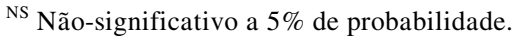

Segundo Stoller \& Sweet (1987), os tubérculos são considerados fator primário de dispersão dessa espécie de planta. Esses pesquisadores afirmam que o comprimento do dia não tem efeito sobre a tuberização, sendo necessária uma temperatura mínima de $20^{\circ} \mathrm{C}$ para estimular o processo. Afirmam também que, mesmo sendo plantas de ciclo $\mathrm{C}_{4}$, sensiveis ao sombreamento, elas conseguem converter de maneira eficiente a matéria seca em tubérculos - Cyperus rotundus converte de 44 a $50 \%$ da sua biomassa seca em tubérculos. Bell et al. (1962) comentam que certos compostos químicos, como etileno, tiouréia, ácido giberélico, éter-etil, benzyladenine, $\mathrm{KSCN}, \mathrm{H}_{2} \mathrm{O}_{2}$ e $\mathrm{O}_{2}$, em determinadas concentrações, podem quebrar a dormência dessas espécies. Esses relatos podem explicar talvez por que, apesar de afetar o número de plantas emersas, a palhada não foi capaz de reduzir a quantidade de tubérculos dessa espécie de planta infestante.

\section{LITERATURA CITADA}

ALMEIDA, F. S. Levantamento da evolução e incidência de plantas daninhas. In: VIEIRA, M. Avaliação de sistemas de cultivo em rotações de culturas. Londrina, IAPAR, 1983. p. 9-11. (Relatório)

ALMEIDA, F. S.; RODRIGUES, B. N.; OLIVEIRA, V. F. Resultados de pesquisa da área de herbologia do IAPAR, da safra de 1982/83. Londrina, IAPAR, 1983. 167 p. (Relatório)

ALMEIDA, F. S.; RODRIGUES, B. N. Guia de herbicidas. Londrina: IAPAR, Livroceres, 1985. 468 p.

BELL, R. S. et al. Life history studies as related to weed control in the Northeast. 1. Nutgrass. 1962. 364 p. (Rhole Island Agric. Exp. Stn. Bull.)

BROWN, P. L.; DICKEY, D. D. Losses of wheat straw residue under simutaled field conditions. Soil Sci. Soc. Am. Proc., v. 34, p. 118-121, 1970.

FENER, M. Germination tests of thirthy-two East African weed species. Weed Res., v. 20, p. 135-338, 1980.

MARTINS, D. et al. Emergência em campo de dicotiledôneas infestantes em solo coberto com palha de cana-de-açúcar. Planta Daninha, v. 17, n. 1, p. 151-161, 1999.

MASON, C. F. Decomposição. São Paulo: EPU/EDUSP, 1980. 63 p. (Temas de Biologia, 18).

PARKER, D. T.; LARSON, W. E. Nitrification as affected by temperature and moisture content of mulched soils. Soil Sci. Soc. Am. Proc., v. 26, p. 238-242, 1962.

STOLLER, E. W.; SWEET, R. D. Biology and life cycle of Purple and Yellow nutsedges (Cyperus rotundus and C. esculentus). Weed Technol., v. 1, p. 66-73, 1987. 
TAYLORSON, R. B.; BORTHWICK, H. A. A. Light filtration by foliar canopies: significance for light-controlled weed seed germination. Weed Sci., v. 17, n. 1, p. 148-151, 1969.

TEDESCO, M. J.; VOLKWEISS, S. J.; BOHNEM, H. Análises de solo, plantas e outras matérias. Porto Alegre: Universidade Federal do Rio Grande do Sul, 1985. 188 p. (Boletim Técnico, 5)
VIGIL, M. F.; KISSEL, D. E. Equations for estimating the amount of nitrogen mineralized from crop residues. Soil Sci. Soc. Am. J., v. 55, p. 757-761, 1998.

WISNIEWSKI, C.; HOLTZ, G. P. Decomposição da palhada e liberação de nitrogênio e fósforo numa rotação aveia-soja sob plantio direto. Pesq. Agropec. Bras., v. 32, n. 11, p. 1191-1197, 1997 